\section{GRP-166 SAFETY EVALUATION OF RITUXIMAB OFF-LABEL USE FOR SYSTEMIC AUTOIMMUNE RHEUMATIC DISEASES}

doi:10.1136/ejhpharm-2013-000276.166

P Pascual, P Carmona, J Barral, M Umerez, B Irastorza, A Aranguren, G Lizeaga, A Asensio, E Esnaola, K Andueza. Donostia University Hospital, Pharmacy Service, San Sebastián, Spain

Background Systemic Autoimmune Rheumatic Diseases (SARDs) are a group of syndromes caused by antibodies inflammation related. Rituximab is a biological drug that targets antigen CD-20 present on the surface of B-Lymphocytes and thus potentially active against SARDs refractories to conventional treatment: steroids and immunosuppressants.

Purpose To describe and evaluate safety parameters of the risk management protocol for adults SARDs patients treated with offlabel Rituximab.

Materials and Methods Descriptive-observational study from January 2011 to July 2012 realised by the Pharmacy and Rheumatology Service. Data were obtained from electronical medical records. Three types of risk management protocol data were evaluated. A) Clinical parameters: infection (including Tuberculosis), cardiovascular disease, severe cytopenia, neoplasia or new neurologic symptoms. B) Complementary tests: hemogram and general biochemistry while on Rituximab. C) Others: adverse events related with Rituximab infusion.

Results 21 patients were included (mean age $52.71 \pm 16.11$ years). Diagnoses were Sjögren's Syndrome (10), Systemic Lupus Erythematosus (4), Mixed Connective Tissue Disease (3), inflammatory myopathy (2), Systemic Sclerosis (1) and Wegener's Granulomatosis (1).

A. Clinical parameters: infection was detected on 5 patients $(23 \%)$, severe cytopenia in 1 patient $(4,7 \%)$ and peripheral neurological symptomatology in another one. Nor cardiovascular disease or neoplasia were detected.

B. Complementary tests: patient presented severe thrombocytopenia (platelets $<2.000 / \mathrm{mcL}$ )

C. Adverse events infusion related: detected on $19 \%$ of patients.

Conclusions Rituximab off-label use for SARDs has increased over the last years and pharmacovigilance strategies as well as risk management protocols have proved useful identifying risks, controlling adverse events, improving quality of care and integrating Pharmacist into direct patient care.

No conflict of interest.

\section{GRP-167 SAFETY OF ADJUVANT CHEMOTHERAPY IN ELDERLY COLON CANCER PATIENTS}

doi:10.1136/ejhpharm-2013-000276.167

'R Aguilella-Vizcaíno, ${ }^{2}$ RM Romero Jiménez, 'P Hidalgo-Collazos, 'T Rico-Gutiérrez, 'R Coloma Peral, 'MT Criado Illana. 'Hospital General de Segovia, Pharmacy, Segovia, Spain; ${ }^{2}$ Hospital General Universitario Gregorio Marañón, Pharmacy, Madrid, Spain

Background Adjuvant chemotherapy trials provide little information on safety in elderly patients because they exclude them or pool their results with those of younger patients.

Purpose To describe the safety of the different adjuvant chemotherapy treatments used in elderly patients with colon cancer.

Materials and Methods Retrospective observational study of colon cancer patients (age >65) diagnosed in 2010 and treated with adjuvant chemotherapy. Each patient was followed from the beginning of the treatment until the end of it. Demographic data, disease stage, antineoplastic agents and treatment-related toxicities were collected from patients' clinical histories.

Results 16 patients ( 5 women, 11 men) were included in the study with a mean age of 75.1 years. $87.5 \%$ and $12.5 \%$ of patients had stage III and stage II disease, respectively. 6 patients (37.5\%) were treated with a combination of 5-fluorouracil and oxaliplatin regimen (FOLFOX), 4 patients (25\%) with capecitabine in monotherapy and the remaining 6 patients $(37.5 \%)$ with a combination of capecitabine and oxaliplatin regimen (XELOX). Adverse events were documented in $100 \%$ of patients. 57 adverse reactions were detected, the most frequent toxicities being: neurotoxicity (75\% of patients), fatigue and anorexia (68.8\%), diarrhoea (37.5\%) and thrombocytopenia (37.5\%). $54.5 \%$ of the undesirable effects were grade $1,30.9 \%$ grade 2 and $14.6 \%$ grade 3 toxicities. There were no grade 4 adverse reactions. XELOX was associated with high rates of hand-foot-syndrome (75\% of patients) and XELOX and FOLFOX with a high incidence of neurotoxicity $(100 \%$ and $83.3 \%$ respectively). Oncologists had to delay the cycle or reduce the treatment doses in 11 patients $(68.8 \%)$ and 5 patients $(31.3 \%)$ had to discontinue the treatment due to the toxicity.

Conclusions A high number of adverse reactions were detected, but majority were grade $1-2$. The safety profile of drugs studied in our population is in line with that described in the literature in younger patients.

No conflict of interest.

\section{GRP-168 SAFETY OF SUNITINIB VERSUS PAZOPANIB IN METASTATIC RENAL CANCER IN A TERTIARY HOSPITAL}

doi:10.1136/ejhpharm-2013-000276.168

M Perez, T Desongles, H Acosta, J Cotrina, M Villalba, M Galván, E Alfaro. Hospital Universitario Virgen del Rocío, Servicio de Farmacia, Seville, Spain

Background Sunitinib and pazopanib are tyrosine kinase inhibitors used in the treatment of metastatic renal cancer. Pazopanib has been approved more recently, so the user experience is not as extensive as with sunitinib.

Purpose To evaluate the safety profile of pazopanib and sunitinib in patients with metastatic renal cancer.

To compare the incidence of adverse reactions between the two drugs.

Materials and Methods We identified patients treated with sunitinib and pazopanib at the hospital in the past two years, using the pharmacy database.

We looked at the medical records of patients through digital medical records, collecting dose patterns, line of therapy, adverse reactions detected, their severity and if dose reductions were necessary, using Excel.

Results A total of 26 patients with metastatic renal cancer were identified: 16 treated with sunitinib and 10 with pazopanib.

Asthenia was the most frequent drug-related toxic effect in both treatment groups, with an incidence of $93.75 \%$ for sunitinib and $60 \%$ for pazopanib.

Nausea/vomiting and diarrhoea were detected in $50 \%$ of patients treated with pazopanib. In sunitinib patients nausea/vomiting were detected in $6.25 \%$ of patients and diarrhoea was detected in $68.75 \%$ of patients

For patients who received pazopanib, the rate of mucositis was $20 \%$, whereas for those treated with sunitinib it was $75 \%$. Palmarplantar erythrodysaesthesia syndrome occurred in $43.75 \%$ of those on sunitinib treatment, while none was detected for pazopanib, and the frequency of other skin pigmentation disorders for the two drugs was $62.5 \%$ and $30 \%$ respectively.

Blood pressure was decompensated in $37.5 \%$ of patients treated with sunitinib and $10 \%$ of those taking pazopanib, although most patients required antihypertensive drugs to get better control.

Dose adjustment was required of sunitinib in $43.75 \%$ of cases and in $25 \%$ pazopanib. 
Conclusions Pazopanib may be better tolerated than sunitinib, with an acceptable adverse event profile and fewer dose adjustments.

Also, the severity of adverse events looks lower with pazopanib.

However, the number of patients was too small to arrive at definitive conclusions, so it is necessary to enlarge this study.

No conflict of interest.

\section{GRP-169 SCREENING FOR CLINICALLY RELEVANT INTERACTIONS IN LIVER TRANSPLANT PATIENTS}

doi:10.1136/ejhpharm-2013-000276.169

${ }^{1} \mathrm{C}$ Andrés González, ${ }^{2} \mathrm{RM}$ Romero Jimenez, ${ }^{3} \mathrm{E}$ Valverde Alcalá, ${ }^{3}$ I Muñoz Castillo. ${ }^{1} H R U$ CARLOS HAYA, pharmacy, Malaga, Spain; 'HRU GREGORIO MARAÑÓN, pharmacy, Madrid, Spain; ${ }^{3}$ HRU CARLOS HAYA, pharmacy, Madrid, Spain

Background Drug-drug interactions are a frequent problem in liver transplant (LT) patients, further hindering pharmacotherapeutic management, which is a very important risk to the patient's life. Purpose To detect drug-drug interaction of clinical relevance in LT patients in a tertiary hospital.

Materials and Methods Descriptive transversal study of the LT patients in our hospital during 2011 who were admitted to the Digestive Surgery Unit (DSU). Variables analysed were: sex, number of drugs prescribed at admission and number of days of hospitalisation in the DSU. Data were collected from clinical and pharmacotherapeutic histories and the unit dose dispensing log. Drug-drug interactions were detected and analysed by the Micromedex Healthcare series ${ }^{\circledR}$ database. The results were analysed with the SPSS v.19 statistics software.

Results Of a total of 51 transplant patients, we included 44 (5 patients died and in 2 patients the medicines were not recorded at admission to the DSU).

$75 \%$ of patients were male and $25 \%$ female, mean age of patients was $53 \pm 12$ years. The median number of days in hospital was 11 [9.18] days. The mean number of drugs prescribed on admission was $11 \pm 2.5 \mathrm{drugs} /$ patient

The total number of drug interactions detected was 210 of which $153(72.9 \%)$ were clinically relevant, representing a prevalence of $84.1 \%$ of liver transplant patients.

Of the main variables studied, only the number of drugs prescribed was found to be directly proportional $(p<0.05)$ to the number of clinically relevant interactions detected, thus no relationship was obtained between age or the number of days hospitalised.

Conclusions Liver transplant patients are critically ill patients with highly complex treatment. A high prevalence of clinically relevant interactions was detected related to polypharmacy and the use of high-risk medicines.

The presence of a pharmacist in this Unit would be beneficial to comprehensively review these patients' treatment.

No conflict of interest.

\section{GRP-170 SEARCHING FOR THE CAUSE OF ALLERGIC CUTANEOUS ADVERSE DRUG REACTIONS: RETROSPECTIVE ANALYSIS OF A FIVE-YEAR CLINICAL EXPLORATION IN A SINGLE- CENTRE COHORT}

doi:10.1136/ejhpharm-2013-000276.170

${ }^{1} \underline{G}$ Maton, ${ }^{2} \mathrm{C}$ Brzezinki, ${ }^{1} \mathrm{H}$ Beaussart, 'D Lannoy, ${ }^{2} \mathrm{D}$ Staumont-Sallé, ${ }^{1} \mathrm{P}$ Odou. ${ }^{1}$ Centre Hospitalier Regional Universitaire, Pharmacy, Lille, France, ${ }^{2}$ Centre Hospitalier Regional Universitaire, Dermatology, Lille, France

Background Adverse drug reactions on skin affect approximately $2 \%$ of patients. Skin and drug challenge tests were performed in the dermatology department to assess these reactions and pharmacy-compounded drugs were tested through patches, pricks and intradermal (IDR) tests.

Purpose To assess the incidence of positive allergic reactions in tested patients and to define the culprit drugs and their potential allergic role in these reactions.

Materials and Methods The study was conducted between 2007 and 2010 on patients from our hospital. We collected information on the characteristics of the adverse drug reaction on skin, the drugs tested, the tests performed and their results.

Results In the period studied, 220 patients referred by other practitioners (from the hospital or from ambulatory practitioners) for serious cutaneous reactions were tested and 3225 preparations were performed by the pharmacy. 92 patients had an immediate reaction to the drug and 128 had a non-immediate reaction. $64(29 \%)$ patients developed a positive response: $48(75 \%)$ through skin tests (patch, prick and IDR) and 16 (25\%) through a Drug Challenge Test (DCT). The drugs most often involved in the positive tests were anti-infectious drugs (46\%), paracetamol (16\%) and iodinated contrast media (10\%).

Conclusions The percentage of positive tests in this cohort agrees with the data found in the literature (3-76\%). The large difference is due to the variability in patient recruitment.

However, it is difficult to compare these data because the preparation and interpretation of the tests are not standardised.

Allergology tests still improve the care of patients as with negative skin tests and DPTs many patients were able to continue with their treatment.

Manufacturing tests by the pharmacy standardise preparation conditions within the hospital and reduce cross contamination and microbial contamination.

No conflict of interest.

\section{GRP-171 SECURING INTRATHECAL INJECTIONS: WHAT ABOUT NON-LUER CONNECTORS?}

doi:10.1136/ejhpharm-2013-000276.171

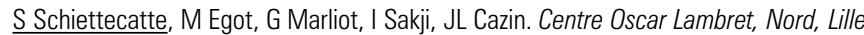
Cedex, France

Background Episodes of accidental injection of medicines intended for intravenous administration into the intrathecal space have been reported worldwide, often leading to death. Since 2001, international guidelines have been issued to prevent such risks. A major recommendation is to develop a non-luer connector to use in neuraxial procedures

Purpose To give an overview of the development and marketing of medical devices fitted with non-luer connectors.

Materials and Methods Manufacturers' catalogues have been consulted. A literature review was conducted using the PubMed and Science Direct databases, including the following MeSH keywords 'non luer', 'connectors', 'safety' and 'intrathecal'. European Health Authorities websites have been also consulted. All searches were performed between August and October 2012.

Results The United Kingdom, which has been a pioneer in guidance, was the first to implement such connectors. Five different non-luer connectors have been designed thanks to the National Patient Safety Agency (NPSA) initiative. Literature research identified few individual tests of these new devices. Some incidents such as mismatching connectors have been documented. So the NPSA has updated recommendations about introducing secure non-luer connectors. These devices are coming onto the French and Belgian market soon. To our knowledge safety connectors are not yet available in other countries.

Conclusions Non-luer connectors for intrathecal drug administration were initially launched in Great Britain. This process obviously 